\title{
Accuracy in Determining Aircraft Position by Terrestrial Radio Navigation
}

\author{
Chingiz Hajiev*, Alper Mehdi Sametoglu². \\ ${ }^{1}$ Istanbul Technical University, Istanbul/Turkey \\ cingiz@itu.edu.tr- (D) 0000-0003-4115-341X \\ ${ }^{2}$ SAJEV Ozel Kucuk Prens High School, Istanbul/Turkey \\ sametoglumehdi@gmail.com- (D) 0000-0001-6289-639X
}

\begin{abstract}
The main objective of terrestrial radio navigation is position determination. In this study, the accuracy of the distance measurement, distance difference measurement, and integrated angle measurement/distance measurement terrestrial radio navigation methods is investigated. in order to calculate the position errors, simulations for the aircraft flight dynamics were carried out, and the obtained position values were compared with the actual values. The aircraft position determination methods were evaluated in the sense of accuracy. The position determination method with better accuracy was determined by comparing the absolute errors of the examined methods. Simulation and error analysis shows that the distance difference method is superior and gives more accurate position results. It was observed that the distance measurement method errors were smaller than the errors of the integrated angle measurement/distance measurement method.
\end{abstract}

\section{Keywords}

\author{
Aircraft \\ Position determination \\ Distance measurement \\ Distance difference measurement \\ Line of positions \\ Terrestrial radio navigation
}

\section{Time Scale of Article}

Received 09 August 2021

Revised until 13 October 2021

Accepted 22 October 2021

Online date 24 December 2021

\section{Introduction}

Terrestrial navigation includes dead reckoning (DR), visual navigation $(\mathrm{VN})$, radio navigation $(\mathrm{RN})$, and some other methods of positioning. These methods are used on land, at sea, and in aviation.

The main objective of terrestrial radio navigation is position determination. Special radio navigation systems provide information on directions, angles, distances, pseudo ranges, and combinations of these types of information.

The radio navigation systems require a technically complex design of a large set of tall antennas and expensive infrastructure (Bhardwaj, 2020). Most radio navigation systems depend on measurement or determining in some way the distance from the known location to the current location of the recipient.
The global terrestrial-based navigation systems include Alpha, developed by the Union of Soviet Socialist Republics (USSR; Alpha, 2020), and Omega, developed by the United States of America (USA) (Omega, 2020). Omega uses the intersection of Line of Positions (LOPs) using distance measurements to determine the user's position. For providing higher accuracy of the receiver, a minimum of three or more independent distance measurements can be used (Bhardwaj, 2020).

LORAN-A (long-range navigation system) is a hyperbolic regional terrestrial-based radio navigation system developed in the USA. This system determines the receiver's position by calculating the time of arrival difference between the signals of the master and slave stations (Bhardwaj, 2020). The regional terrestrial-based navigation system LORAN-B, developed in the United States, provides an accuracy of the order of several tens of feet (LORAN, 2020).

\footnotetext{
*: Corresponding Author Chingiz Hajiev, cingiz@itu.edu.tr

DOI: 10.23890/IJAST.vm02is02.0201
} 
The proposal to transition from terrestrial-based navigation aids to satellite and aerial surveillance as a primary means of navigation is presented (Blakey, 2006). From this point on, the Global Positioning System (GPS) is considered the main navigation method in terrestrial and oceanic travel.

LORAN-C is the terrestrial-based navigation system which is developed by the USA (LORAN, 2020). Loran-C is a more successful system, and attempts are being made to integrate into solution positioning, navigation, and timing (PNT) services together with satellite navigation systems. LORAN-C support can correct the global navigation satellite systems (GNSS) uncertainties against jamming (Gluch et al., 2000).

In terrestrial radio navigation, a VHF omnidirectional range (VOR) and distance measuring equipment (DME) are used widely. The VOR allows the receiver to measure its bearing to or from the beacon. DME is defined as a navigation beacon to measure the aircraft position relative to that beacon. Aircraft send out a signal which is sent back after a fixed delay by the DME hardware. The aircraft can compute its distance to the beacon from the delay of the signal received by the DME instruments (Krinetskiy 1977; Haciyev, 1999).

An aircraft's position in space is defined by three coordinates. In radio navigation, for aircraft's position characteristics, the surface of positions (SOPs) and line of positions are used. The aircraft's position is defined by two position lines or three-position surfaces or by position line and position surface. As a navigation parameter, elevation angle, azimuth angle, distance, distance difference, or sum of distances can be used (Krinetskiy 1977; Kayton and Fried, 1997).

In general, when three geometric surfaces cross, there are a few intersecting points (Hajiyev, 1999). In this case, there may be several solutions for determining the aircraft's position. This problem can be avoided by using extra information for each position.

The error analysis in determining aircraft coordinates by the distance measurement method is given in (Haciyev and Üner, 1998). In (Erkal and Haciyev, 2004), the accuracy of the distance difference measurement method is investigated. However, these studies do not compare different types of terrestrial radio navigation techniques.

In this study, the accuracy of the distance measurement, distance difference measurement, and integrated angle measurement/distance measurement terrestrial radio navigation methods is investigated using simulations. For this purpose, the aircraft flight dynamics are simulated, and the obtained values of the coordinates were compared with the actual values. The aircraft position determination method with better accuracy was determined by comparing the absolute errors of the examined methods.

\section{Mathematical Model of Aircraft Dynamics}

The mathematical model of the aircraft's motion consists of longitudinal and lateral motion models. The longitudinal motion of the aircraft consists of forwarding, vertical, and pitching motions. The state vector $\mathrm{X}_{\mathrm{u}}$ and control vector $\mathrm{U}_{\mathrm{u}}$ of the longitudinal model can be written in the following form:

$$
\begin{gathered}
X_{u}=\left[\begin{array}{cccc}
u & w & q & \theta
\end{array}\right]^{T} \\
u=\left[\begin{array}{l}
\delta_{E}
\end{array}\right]
\end{gathered}
$$

where

$\mathrm{u}$ : forward velocity of the aircraft $(\mathrm{m} / \mathrm{s})$

w : vertical velocity of the aircraft $(\mathrm{m} / \mathrm{s})$

$\mathrm{q} \quad$ : pitching rate of the aircraft (degree/s)

$\theta \quad$ : pitching angle of the aircraft (degree)

$\delta_{E} \quad$ : elevator deflection (degree)

The mathematical model of the longitudinal motion is given by the following differential equation,

$$
\dot{X}_{u}=A_{u} X_{u}+B_{u} U_{u}
$$

where $\mathrm{Au}$ is the system matrix, $\mathrm{Bu}$ is the control distribution matrix. These matrices are (McLean, 1990):

$$
\begin{aligned}
A_{u} & =\left[\begin{array}{cccc}
X_{u} & X_{w} & 0 & -g \cos \gamma_{0} \\
Z_{u} & Z_{w} & U_{0} & -g \sin \gamma_{0} \\
\widetilde{M}_{u} & \widetilde{M} & \widetilde{M} & \widetilde{M}_{\theta} \\
0 & 0 & 1 & 0
\end{array}\right] \\
B_{u} & =\left[\begin{array}{c}
X_{\delta_{E}} \\
Z_{\delta_{E}} \\
\widetilde{M}_{\delta_{E}} \\
0
\end{array}\right]
\end{aligned}
$$

The lateral motion of the aircraft consists of sideslip, roll, and yaw motions. The state vector $X_{y}$ and control vector $U_{y}$ of the lateral model can be written as:

$$
\begin{gathered}
X_{y}=\left[\begin{array}{lllll}
\beta & p & r & \varphi & \psi
\end{array}\right]^{T} \\
u=\left[\begin{array}{l}
\delta_{A} \\
\delta_{R}
\end{array}\right]
\end{gathered}
$$

where

$\beta \quad$ : sideslip angle of the aircraft (degree)

$p \quad$ : rolling rate of the aircraft (degree/s)

$r$ : yawing rate of the aircraft (degree/s)

$\varphi \quad$ : rolling angle of the aircraft (degree) 
$\psi \quad$ : yawing angle of the aircraft (degree)

$\delta_{A} \quad$ : aileron deflection angle (degree)

$\delta_{R} \quad$ : rudder deflection angle (degree)

The mathematical model of the lateral motion is given by

$$
\dot{Z}_{y}=A_{y} X_{y}+B_{y} U_{y}
$$

where the system matrix Ay and the control distribution matrix by are (McLean, 1990):

$$
\begin{aligned}
A_{y} & =\left[\begin{array}{ccccc}
Y_{v} & 0 & U_{0} & g \cos _{\gamma_{0}} & 0 \\
L_{v}^{\prime} & L_{p}^{\prime} & L_{r}^{\prime} & 0 & 0 \\
N_{v}^{\prime} & N_{p}^{\prime} & N_{r}^{\prime} & 0 & 0 \\
0 & 1 & \tan _{\gamma_{0}} & 0 & 0 \\
0 & 0 & \sec _{\gamma_{0}} & 0 & 0
\end{array}\right] \\
B_{y} & =\left[\begin{array}{cc}
0 & Y_{\delta_{R}} \\
L_{\delta_{A}}^{\prime} & L_{\delta_{R}}^{\prime} \\
N_{\delta_{A}}^{\prime} & N_{\delta_{R}}^{\prime} \\
0 & 0 \\
0 & 0
\end{array}\right]
\end{aligned}
$$

For small side slip velocities, $\mathrm{v}=\mathrm{U}_{0} \beta$ assumption can be made. Notations in (2), (3), (5), and (6) can be found in (McLean, 1990).

Additional expressions must be included in the model to determine the positions along $X, Y$ and $Z$ directions:

$$
\begin{aligned}
& \dot{x}=u \\
& \dot{y}=\mathrm{v}=U_{0} \beta \\
& \dot{z}=w
\end{aligned}
$$

The state vector of the mathematical model of aircraft is,

$$
X=\left[\begin{array}{llllllllllll}
u & w & q & \theta & \beta & p & r & \phi & \psi & x & y & z
\end{array}\right]^{T}
$$

The control vector of the system is,

$$
U=\left[\delta_{E} \delta_{A} \delta_{R}\right]^{T}
$$

Discretizing the aircraft's mathematical model, we have

$$
\begin{aligned}
& X=A X+B U \Rightarrow X_{i}=\frac{X_{\mathrm{I}+1}-X_{\mathrm{I}}}{\Delta t}=A X_{i}+B U_{i}, \\
& X_{i+1}-X_{\mathrm{I}}=A \Delta t . X+B \Delta t \cdot u_{i}, \\
& X_{i+1}=\underbrace{(I+\Delta t A)}_{A^{*}} X_{i}+\underbrace{\Delta t \cdot B U_{i}}_{B^{*}} \Rightarrow \\
& X_{i+1}=A^{*} X_{i}+B^{*} U_{i}
\end{aligned}
$$

where $X$ is the aircraft state vector, $A^{*}$ is the system matrix, $U$ is the control input vector, $B^{*}$ is the control distribution matrix

\section{Determination of Aircraft's Coordinates Via}

\section{Distance Measurement Method}

In the distance measurement method, the position surface is a sphere with radius D which is the distance from the station to the aircraft. Thus, an aircraft's position in a space can be found by intersecting three $\mathrm{D}=$ const position surfaces. The intersection of the three spheres is shown in Figure 1.

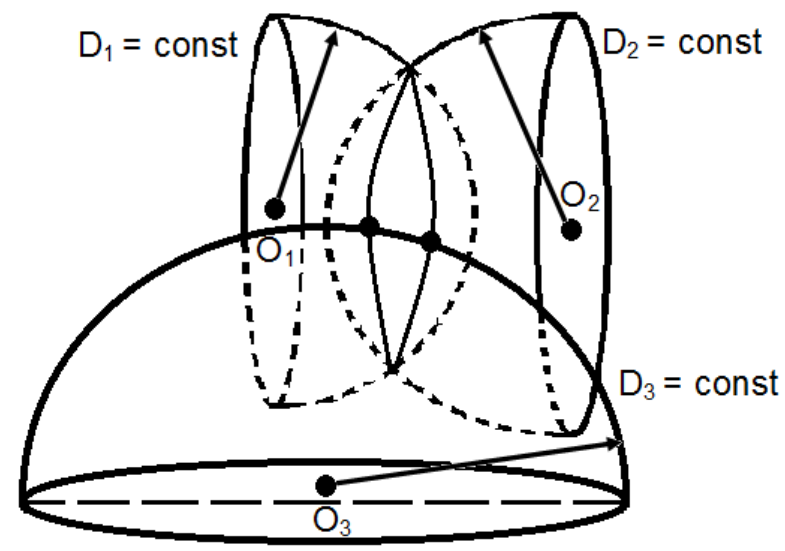

Fig.1. The intersection of three spheres

Since there are two intersection points when intersecting three spheres, the results of this method are uncertain. To make this method more precise, it can be integrated with extra navigation systems with low accuracy.

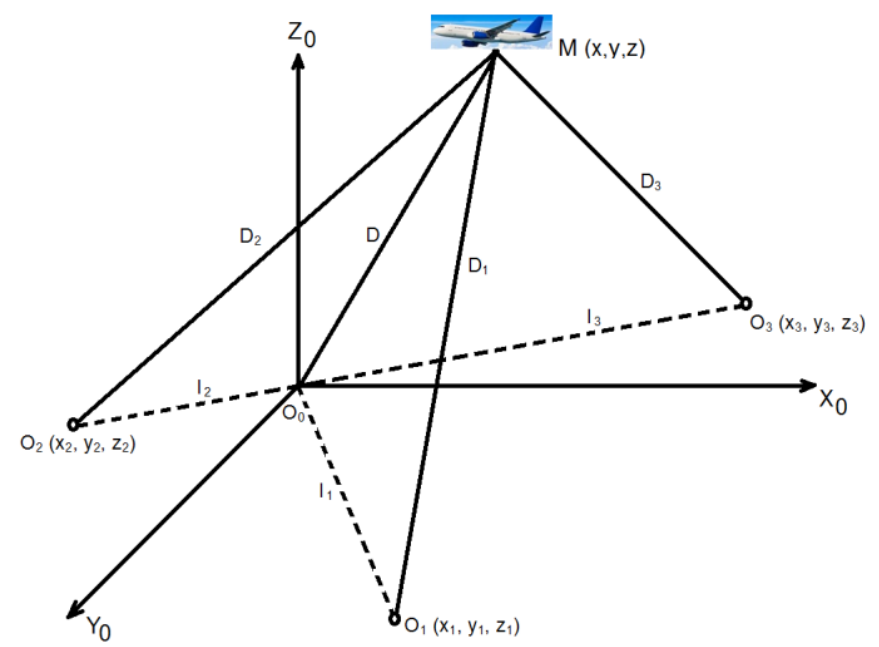

Fig. 2. Distance measurement method's scheme in determining the position of the aircraft

Three measurement stations (distance measurement stations) are located on the ground to determine the aircraft's position. Let us assume that the ground stations located on $\mathrm{O}_{1}\left(x_{1}, y_{1}, z_{1}\right), \mathrm{O}_{2}\left(x_{2}, y_{2}, z_{2}\right)$, and $\mathrm{O}_{3}\left(x_{3}, y_{3}, z_{3}\right)$ points of $\mathrm{O}_{0} \mathrm{X}_{0} \mathrm{Y}_{0} \mathrm{Z}_{0}$ Descartes coordinate system to find calculation formulas of distance measurement method (See Fig. 2). $l_{1}, l_{2}$ and $l_{3}$ show the distance between the ground station's origins and the origin of $\mathrm{O}$ ${ }_{0} \mathrm{X}_{0} \mathrm{Y}_{0} \mathrm{Z}_{0}$ coordinate system, and $\mathrm{D}_{1}, \mathrm{D}_{2}$, and $\mathrm{D}_{3}$ show the distances to the aircraft at $M(x, y, z)$ point. The equations for the coordinate determination can be written as (Haciyev, 1999): 


$$
\begin{aligned}
& \left(x_{1}-x_{2}\right) x+\left(y_{1}-y_{2}\right) y+\left(z_{1}-z_{2}\right) z=c_{1} \\
& \left(x_{1}-x_{3}\right) x+\left(y_{1}-y_{3}\right) y+\left(z_{1}-z_{3}\right) z=c_{2} \\
& \left(x_{2}-x_{3}\right) x+\left(y_{2}-y_{3}\right) y+\left(z_{2}-z_{3}\right) z=c_{3}
\end{aligned}
$$

Here

$$
\begin{aligned}
& c_{1}=\frac{1}{2}\left(D_{2}^{2}-D_{1}^{2}+l_{1}^{2}-l_{2}^{2}\right) \\
& c_{2}=\frac{1}{2}\left(D_{3}^{2}-D_{1}^{2}+l_{1}^{2}-l_{3}^{2}\right) \\
& c_{3}=\frac{1}{2}\left(D_{3}^{2}-D_{2}^{2}+l_{2}^{2}-l_{3}^{2}\right)
\end{aligned}
$$

The solution of equations (11)-(13) for aircraft coordinate determination can be found as follows (Sametoğlu and Hajiyev 2021):

$$
\begin{aligned}
& x=e_{1}-a z \\
& y=e_{2}-b z \\
& \left(a^{2}+b^{2}+1\right) z^{2}+2\left(x_{1} a-z_{1}+y_{1} b-a e_{1}-b e_{2}\right) z- \\
& \left(D_{1}^{2}-l_{1}^{2}-e_{1}^{2}-e_{2}^{2}+2 x_{1} e_{2}\right)=0
\end{aligned}
$$

where

$$
\begin{aligned}
& e_{1}=\frac{c_{1}\left(y_{2}-y_{3}\right)-c_{3}\left(y_{1}-y_{2}\right)}{\left(x_{1}-x_{2}\right)\left(y_{2}-y\right)-\left(x_{2}-x_{3}\right)\left(y_{1}-y\right)} ; \\
& e_{2}=\frac{c_{1}\left(x_{1}-x_{3}\right)-c_{3}\left(y_{1}-y_{2}\right)}{\left(x_{1}-x_{2}\right)\left(y_{1}-y\right)-\left(x_{1}-x_{2}\right)\left(y_{1}-y_{3}\right)} ; \\
& \mathrm{a}=\frac{\left(\mathrm{z}-z_{2}\right)\left(y_{2}-y\right)-\left(z_{2}-\mathrm{z}\right)\left(y_{1}-y\right)}{\left(x_{1}-x_{2}\right)\left(y_{2}-y\right)-\left(x_{2}-x_{3}\right)\left(y_{1}-y\right)} ; \\
& \mathrm{b}=\frac{\left(z_{1}-z_{2}\right)\left(x_{2}-x_{3}\right)-\left(x_{1}-z\right)\left(x_{1}-x_{2}\right)}{\left(x_{1}-x_{3}\right)\left(y_{1}-y_{2}\right)-\left(x_{1}-x_{2}\right)\left(y_{1}-y\right)}
\end{aligned}
$$

\section{Determination of Aircraft Coordinates Via}

\section{Distance Difference Measurement Method}

In distance difference measurement, radio-navigation systems, the distances from two ground stations to aircraft are taken as navigation parameters (Krinetskiy 1979). Measurement of $\Delta \mathrm{D}$ distance differences allow to determine that the aircraft locates on $\Delta \mathrm{D}=$ constant state surface. This surface is in the form of rotating hyperboloids of the two-leaf stage, which has measurement stations on $\mathrm{O}$ and $\mathrm{O}_{1}$ centers (Figure 3).

The aircraft's position is found as the intersection point of three state surfaces. Therefore, the distancedifference measurement radio-navigation system includes four ground stations. One of these stations is the router (main station), and the others are directed (assistant stations). With the help of signals sent by the main station, three directed stations are provided to work synchronously. With the distance-difference method, we derive the following expressions and get $x, y$ and $z$ coordinates of an aircraft:

$$
\begin{aligned}
& x_{1} x+y_{1} y+z_{1} z-D \Delta D_{1}=f_{1} \\
& x_{2} x+y_{2} y+z_{2} z-D \Delta D_{2}=f_{2}
\end{aligned}
$$

$$
x_{3} x+y_{3} y+z_{3} z-D \Delta D_{3}=f_{3}
$$

here,

$$
\begin{aligned}
& \Delta D_{1}=D-D_{1} \\
& \Delta D_{2}=D-D_{2} \\
& \Delta D_{3}=D-D_{3}
\end{aligned}
$$

are the range differences.

$$
\begin{aligned}
& f_{1}=\frac{1}{2}\left(l_{1}^{2}-\Delta D_{1}^{2}\right) \\
& f_{2}=\frac{1}{2}\left(l_{2}^{2}-\Delta D_{2}^{2}\right) \\
& f_{3}=\frac{1}{2}\left(l_{3}^{2}-\Delta D_{3}^{2}\right)
\end{aligned}
$$

Via solving (24) - (26) equations, the expressions required for the aircraft coordinates $x, y, z$ are found.

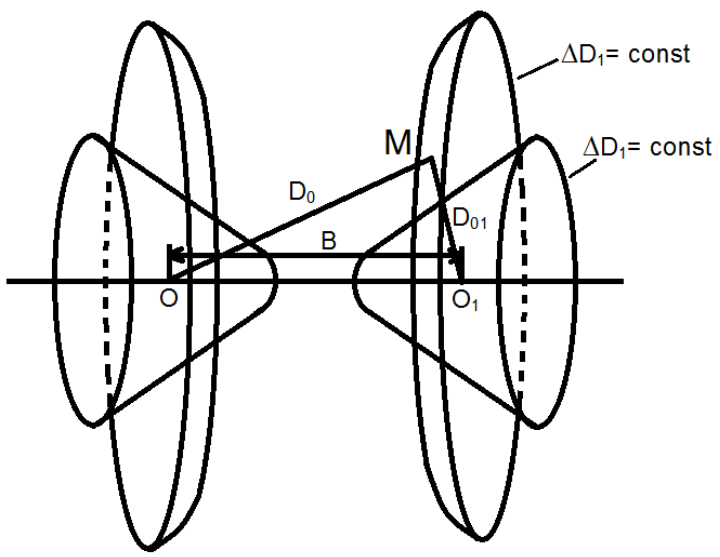

Fig.3. Illustration of the state surfaces in the distance difference measurement method.

\section{Determination of Aircraft's Coordinates by}

\section{Integrated Method}

Measurement of angle and measurement of distance methods are used at the same time in the integrated method (Haciyev, 1999). The integrated method is usually used in radiolocation systems, and it determines $\mathrm{D}$ distance to the aircraft, azimuth angle $\alpha$, and elevation angle $\beta$. When this method is used, the coordinates of the aircraft are determined as the intersection point of the sphere state surface $(D=$ constant $)$, cone state surface $(\beta=$ constant $)$, and vertical plane suitable for $\alpha=$ constant state surface (Fig. 4). The aircraft coordinates are determined with a single point (ground station) with the help of this method without the need for difficult calculations.

Following formulas are used to calculate aircraft coordinates;

$$
\begin{aligned}
& x=D \cos \beta \cos a \\
& y=D \cos \beta \sin a \\
& z=D \sin \beta
\end{aligned}
$$


Distance, azimuth, and elevation angles are determined by radiolocation measurements. A single radiolocation station is enough for this method

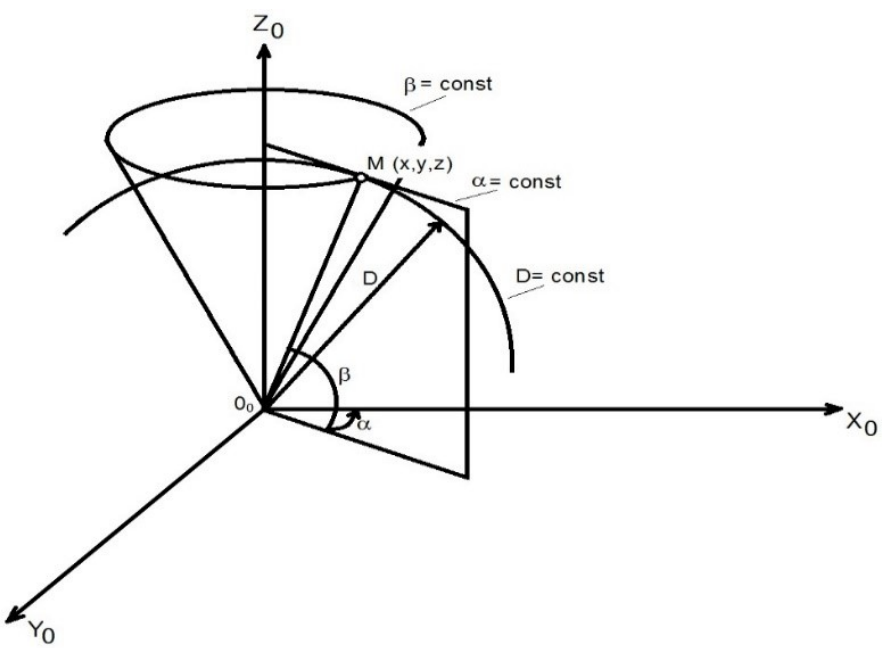

Fig.4. Illustration of the state surfaces in the integrated method.

\section{Simulation Results and Discussion}

The distance measurements are simulated via the equation below:

$$
\begin{aligned}
& D_{z_{i}}=\sqrt{\left(x_{i}-x\right)^{2}+\left(y_{i}-y\right)^{2}+\left(z_{i}-z\right)^{2}} \\
& +b+\sigma_{D} \text { randn }
\end{aligned}
$$

where $x_{i}, y_{i}$ and $z_{i}$ are the coordinates of the ground radio-navigation stations, $b$ is the bias in the distance measurements, $\sigma_{D}$ is the standard deviation of random distance measurement error, which is $\sigma_{D}=10 \mathrm{~m}$ in simulations.

The simulations are performed for the azimuth angle $\alpha$ and elevation angle $\beta$ via the expressions below;

$$
\begin{aligned}
& a=\operatorname{ArcTan}\left[\frac{Y}{X}\right] ; \\
& \beta=\operatorname{ArcTan}\left[\frac{Z}{X} \cos a\right]
\end{aligned}
$$

Matlab software was used to investigate the accuracy of terrestrial radio navigation methods through simulations.

The simulation results and error analysis show that the distance difference method gives more accurate position results. The absolute errors of the distancedifference measurement method along the $\mathrm{x}, \mathrm{y}$, and $\mathrm{z}$ axes are given in Fig. 5, 6, and 7, respectively.

The aircraft position error analysis led to the result that the distance difference method is more accurate for horizontal $\mathrm{x}$ and $\mathrm{y}$ coordinates while it is not as much as good for the vertical $z$ coordinate. As seen from the graphs, the absolute errors of horizontal $\mathrm{x}$ and $\mathrm{y}$ coordinates are good and varies approximately within the limits of $[-25 ;+25]$ meters. The disadvantage of this method is that 4 ground stations are required for implementation.

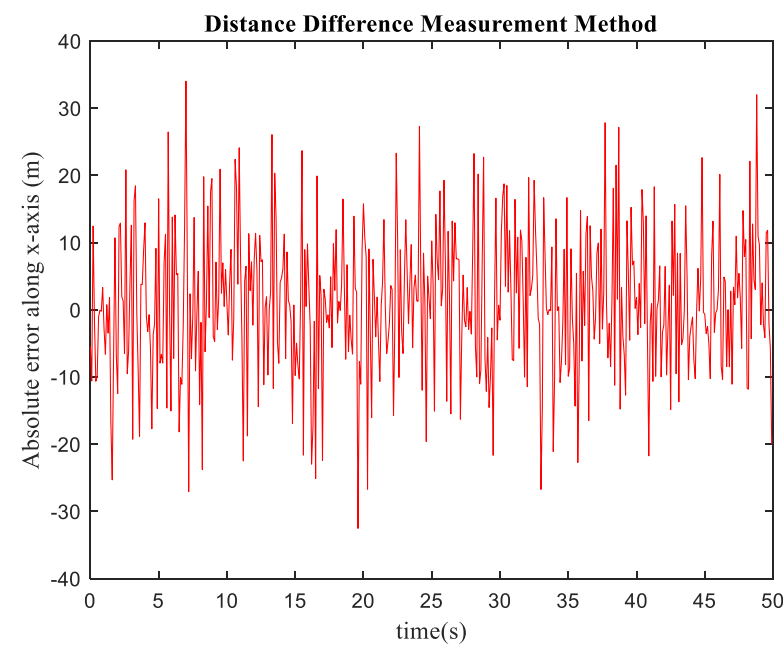

Fig. 5. Absolute error along the $x$-axis when using the distance difference measurement method.

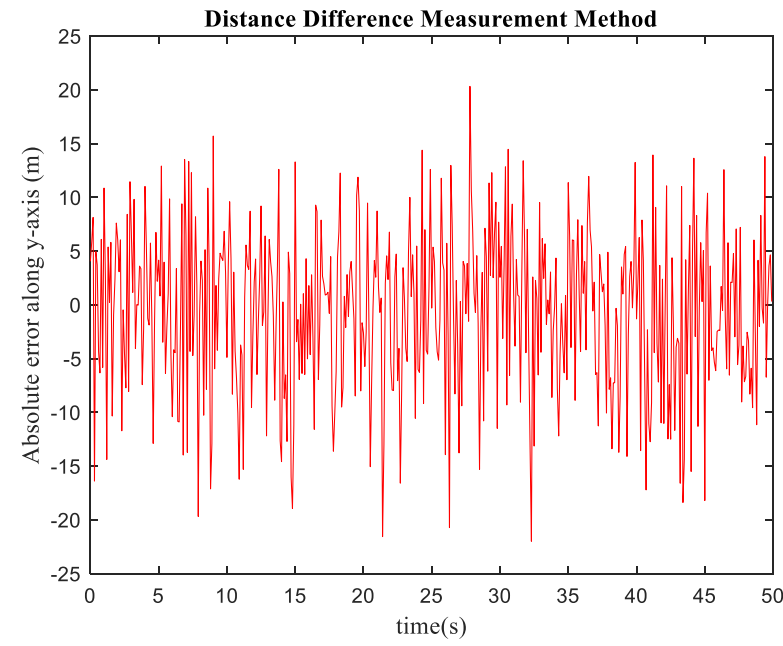

Fig.6. Absolute error along the y-axis when using the distance difference measurement method.

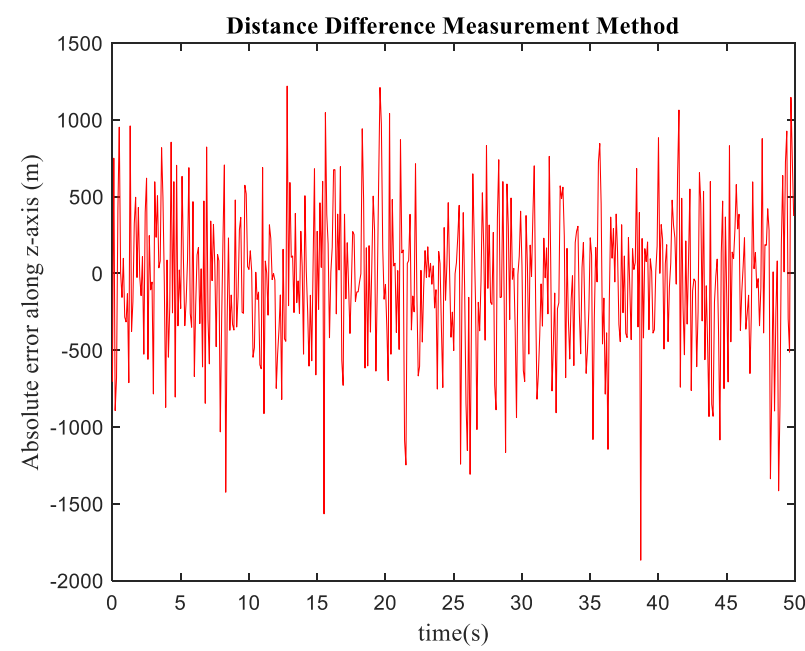

Fig.7. Absolute error along the $\mathrm{z}$-axis when using the distance difference measurement method. 
The relative errors of the distance-difference measurement method along the $\mathrm{x}, \mathrm{y}$ and $\mathrm{z}$ axes are presented in Figs. 8, 9, and 10, respectively.

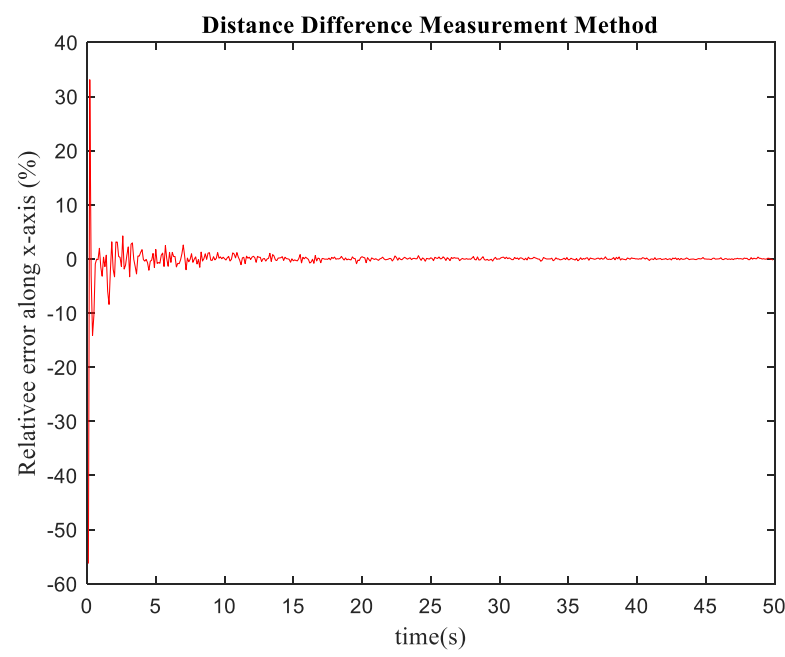

Fig. 8. Relative error along the $\mathrm{x}$-axis when using the distance difference measurement method.

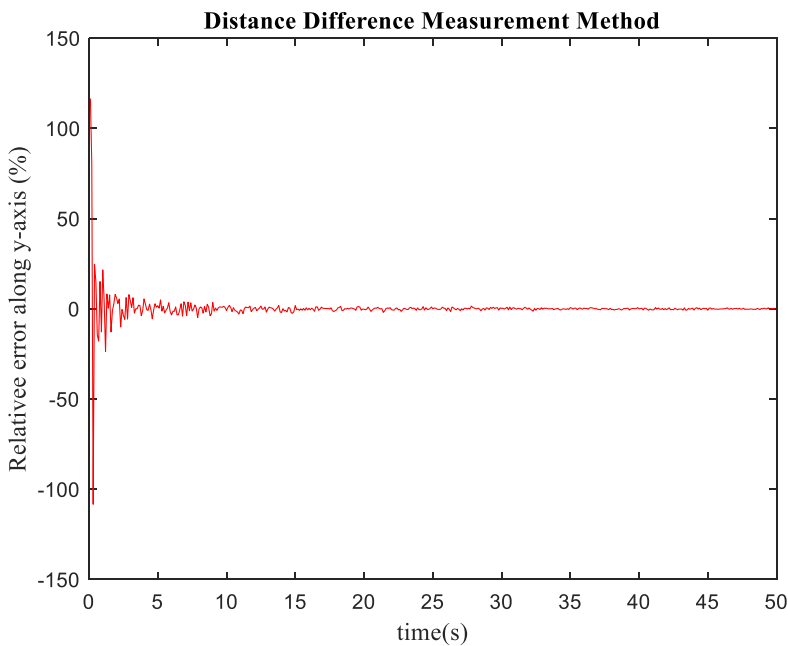

Fig. 9. Relative error along the y-axis when using the distance difference measurement method.

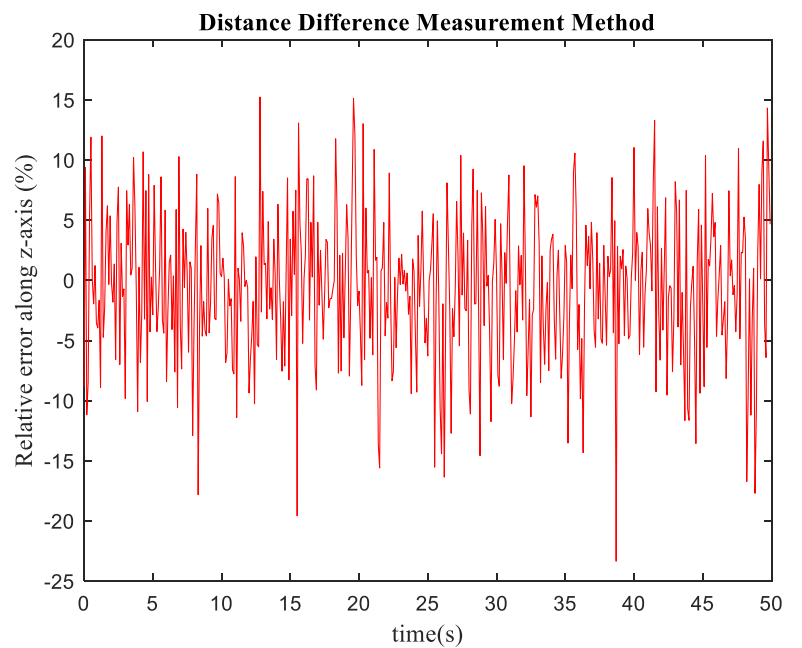

Fig. 10. Relative error along the $z$-axis when using the distance difference measurement method.
The results shown in Figures 8, 9, and 10 show that the relative errors in the $\mathrm{x}$ and $\mathrm{y}$ coordinates are good enough but high on the $\mathrm{z}$-axis.

Simulation results are also obtained for distance measurement and integrated methods. The obtained results show that the errors calculated in the distance measurement method are smaller than the errors found in the integrated angle measurement/distance measurement method. As a result, calculating the aircraft coordinates with the distance measurement method is more advantageous than the integrated method. However, to implement the distance measurement method, three ground stations are required, whereas only one station is necessary for the integrated method.

The integrated method requires only one ground station, but the position determination accuracy deteriorates as the aircraft moves away from that station. The simulation results presented in Figure 11, showing absolute errors along the $y$-axis when using the integrated method, confirm this statement.

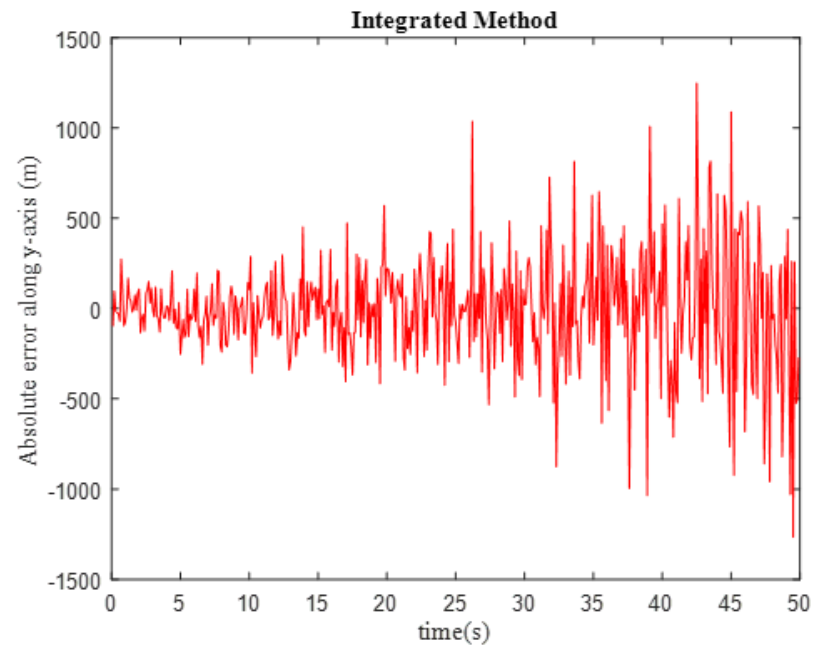

Fig. 11. Absolute error along the $y$-axis when using the integrated method.

\section{Combination of Terrestrial and Satellite-Based}

\section{Navigation Systems}

The study (Bhardwaj, 2020) shows that there is a large potential for the combined navigation solutions from terrestrial and Satellite-based navigation systems.

In the case of satellite navigation methods, the main limitation arises in closed areas such as dense forest and tunnel areas or underground areas where local solutions such as radio frequency identification (RFID) or wireless local area networks (LANs) provide indoor location tracking systems are available (Chothani et al., 2015). Thus, it is necessary to combine the two methods and provide a more reliable system, overcoming the 
limitations of existing terrestrial and satellite navigation systems (Bhardwaj, 2020).

Recently developed regional systems include the QuasiZenith Satellite System (QZSS) developed in Japan and the Indian Regional Navigation Satellite System (IRNSS).

Global and regional satellite navigation systems provide their services with an accuracy of about 10-20 meters. A literature review presented in (Bhardwaj, 2020) shows that regional satellite navigation systems such as IRNSS and QZSS provide accuracy comparable to GNSS in their main service region.

Terrestrial radio navigation systems can include the same kinds of faults as GNSS. The terrestrial radio signals can be subject to multipath, while interference or signal attenuation can affect all or some signals at a given location. These problems can cause many receivers to produce a stream of false measurements prior to detecting signal unavailability. The user equipment can also include hardware or software failures (Groves, 2008).

\section{Conclusion}

In this study, the accuracy of the different terrestrial radio navigation methods is investigated. The distance measurement, distance difference measurement, and integrated angle measurement/distance measurement aircraft position determination methods are taken into consideration. Simulations were carried out for aircraft flight dynamics. The position values that are determined by the presented methods were compared with the actual values.

Simulation and error analysis led to the result that the distance difference method is superior and gives more accurate position results. It was observed that the distance measurement method errors were smaller than the errors of the integrated method.

The demonstrations also show that the examined aircraft position determination methods are more accurate in determining the horizontal $\mathrm{x}$ and $\mathrm{y}$ coordinates than the vertical $\mathrm{z}$ coordinate determination.

There is a good potential for the combined navigation solutions of terrestrial and satellite-based navigation systems.

\section{CRediT Author Statement}

Chingiz Hajiyev: Conceptualization, Methodology, Investigation, Validation, Supervision, Writing-Original Draft. Alper Mehdi Sametoğlu: Writing-Review, Investigation, Software, Visualization.

\section{Abbreviations}

\begin{tabular}{ll}
\hline DME & : Distance measuring equipment \\
DR & : Dead Reckoning \\
GNSS & : Global Navigation Satellite Systems \\
GPS & : Global Positioning System \\
IRNSS & : Indian Regional Navigation Satellite System \\
LANs & : Local Area Networks \\
LOPs & : Line of Positions \\
LORAN & : Long-Range Navigation System \\
PNT & : Positioning, Navigation and Timing \\
QZSS & : Quasi-Zenith Satellite System \\
RFID & $:$ Radio-Frequency Identification \\
RN & : Radio Navigation \\
SOPs & $:$ Surface of Positions \\
USA & : United States of America \\
USSA & : Union of Soviet Socialist Republics \\
VN & : Visual Navigation \\
VOR & $:$ Omnidirectional range \\
\hline
\end{tabular}

\section{References}

Alpha (Navigation), Available at: https://en.wikipedia.org/wiki/Alpha_(navigation) (accessed 14 October 2020).

Bhardwaj A., 2020, Terrestrial and Satellite-Based Positioning and Navigation Systems-A Review with a Regional and Global Perspective, Engineering Proceedings, 2, 41, 2-7.

Blakey, J.H., 2006, Navigating Towards the Future: Transitioning from Terrestrial Radio Navigation to Satellite Navigation and Airborne Surveillance, IEEE Aerospace \& Electronic Systems Magazine, May, 1721.

Chothani, A., Saindane, J., Mistari, H., Bhavsar, N., Shirsath, R., 2015, RFID-based location tracking system using a RSS and da, Proc. of the International Conference on Energy Systems and Applications (ICESA 2015), 30 October-1 November 2015, Pune, India, pp. 748-751.

Erkal, Y. and Ç. Hacıyev, 2004, Determination of aircraft motion parameters using GNSS based on distance difference measurement and Doppler methods and error analysis, Aviation Advanced Technologies and Applications Symposium, Vol.1, December 9-10, 2004, Istanbul, Turkey.

Gluch, M., Kirchner, J. Herzig, M., 2000, Development of Software Structure for Integrated Navigation Receivers in Shipping. IFAC Proc., 33, pp. 407-412.

Groves, P.D.,2008, Principles of GNSS, Inertial, and Multisensor Integrated Navigation Systems. Boston; London: Artech House. 
Haciyev, Ç.M., 1999, Radyo Navigasyon (Radio Navigation), 1st edition, Istanbul Technical University, Istanbul.

Hacıyev, Ç. and Ü. Üner, 1998, Guaranteed error analysis in determining the coordinates of aircraft by measuring distances, 2nd Aeronautical Symposium, May 11-15, 1998, Kayseri, Turkey.

M. Kayton, \& W. R. Fried (1997). Avionics navigation systems. John Wiley \& Sons.

Krinetskiy Ye.I. (ed.), 1979, Flight Tests of Rockets and Space Apparatus. Moscow: Maşinostroyeniye (in Russian).

LORAN, Available at: https://en.wikipedia.org/wiki/LORAN, (available accessed 12 October 2020).

McLean, D., 1990, Automatic Flight Control Systems. Hertfordshire, UK: Prentice Hall International.

Omega (Navigation System). Available online: https://en.wikipedia.org/wiki/Omega_(navigatio n_system) (accessed on 14 October 2020).

Sametoğlu, A.M. and Hajiyev, C., 2021, Investigation of the accuracy of terrestrial radio navigation methods for determining aircraft position, International Symposium on Aircraft Technology, MRO \& Operations, June 28-30, 2021, Budapest, Hungary. 